\title{
Neurocognitive mechanisms of prosociality in childhood
}

Steinbeis, Nikolaus

Department of Developmental and Educational Psychology

University of Leiden

Wassenaarseweg 54

2333 AK Leiden

Netherlands

Word Count: 1975

Running Head: On the neural bases of prosociality in childhood

Key words: Prosocial Behavior, Sharing, Helping, Behavioral Control, Emotion Regulation, DLPFC, ACC, Insula 


\section{Abstract}

This paper reviews the neurocognitive mechanisms underlying prosocial development in childhood. I begin by arguing that most prosociality is costly. This cost needs to be regulated for prosocial behavior to occur. The precise regulatory mechanisms depend on the type of prosocial behavior and include behavioral control in the case of sharing and emotion regulation in the case of helping. I review evidence that these regulatory mechanisms are subserved by prefrontal cortical circuitry, which depending on the mechanism interacts with different brain regions coding for self- and other-related affect to produce prosocial behavior. I conclude that the maturation of prefrontal cortical circuitry drives the development of both sharing and helping in childhood through supporting the emergence of relevant regulatory mechanisms. 


\section{Introduction}

Prosocial behavior can take multiple forms, such as sharing resources (i.e. food or money), helping, or comforting others in distress. These forms of prosociality have been documented already in infancy [1], suggesting early and deep-seated ontogenetic roots [2]. While much attention has been dedicated to understanding the origins of prosociality, less research has been conducted on how these behaviors develop throughout childhood and into adulthood. This paper reviews studies on the neurocognitive mechanisms of prosocial development, specifically sharing and helping, during middle and older childhood ${ }^{1}$.

I argue that most types of prosocial behavior, and certainly sharing and helping carry a cost. Sharing resources means less for oneself and helping another requires time and physical or psychological effort. At the very least prosocial behavior implies opportunity costs with regards to both resource and recipient. Studies have shown that children are aware of these costs since, their prosocial behavior is modulated by both the potentially incurred cost $[3,4]$ and the value of the resource [5]. I propose that the development of prosociality can be viewed through a value-based decision-making framework [6], whereby the potential costs of prosociality are weighed up against the possible benefits derived from being prosocial, both in the short- and the long-term. I argue that for decisions to be swayed in favour of prosociality, the costs of being prosocial need to be regulated. These regulatory processes undergo protracted developmental

\footnotetext{
1 The literature on neural correlates of comforting in children and adults is virtually non-existent.
} 
change due to the maturation of the underlying neural circuitry. This paper reviews studies showing that distinct forms of prosociality demand distinct regulatory processes. Thus, sharing needs behavioral control and helping requires emotion regulating. These regulatory processes draw on distinct neural mechanisms, which might also explain the observed lack of positive relationships between the two types of prosociality in development $[1,7]$.

\section{The neurocognitive mechanisms of sharing}

Sharing valuable resources can be observed in toddlers as young as 15 months [8]. At this age sensitivity towards equal distributions and fairness norms also emerges $[9,10]$. Around 3 years, children state that sharing equally is the norm and from then on, they increasingly follow such sharing norms with their actual behavior [11]. Complying with social norms constitutes a long-term goal, which conflicts with the more immediate satisfaction of reward maximization [12]. I argue that resolving such conflict in favour of sharing according to the norm requires behavioral control. There is by now increasing evidence that behavioral control is positively correlated to sharing in both preschoolers [13, 14] and school children $[11,15]$ using a variety of measures of behavioral inhibition (i.e. day-night task, questionnaire measures). More recently, these correlative findings have been extended to show that explicit experimental manipulations of behavioral control impact sharing directly. Thus, in one study, it was shown that children aged 6-9 years shared less after having engaged in a behavioral motor control task compared to sharing after a speeded reaction time task [16]. In another study children aged 6-9 years shared more after having listened to stories priming behavioral control compared to stories that did not [17]. In sum, 
there is a sizeable body of literature suggesting that behavioral control aids sharing in accordance with the prevailing social norms during middle childhood.

In adults, it has been shown that social norm compliance relies on lateral prefrontal cortical brain regions $[18,19]$. Activity in left and right dorsolateral prefrontal cortex (DLPFC, see Figure 1A) was positively correlated with sharing under threat of punishment [18], while disrupting activity in right DLPFC reduced such sharing [19]. It has been argued that the top-down modulation from DLPFC of subjective value-signals in the ventromedial prefrontal cortex (VMPFC, see Figure 1B) is key for both implementing and complying with social norms [20]. Lateral prefrontal cortical areas are among the brain regions undergoing the most protracted age-related loss of grey matter volume throughout childhood and adolescence [21]. Further, linear age-related increases in structural connectivity are among the most delayed in white matter bordering prefrontal cortex [22], which in turn impacts the extent of functional connectivity [23]. Lateral portions of the prefrontal cortex are involved in actively maintaining task goals, biasing attention and implementing behaviors [24]. The maturation of lateral PFC also underpins the development of these functions in children $[25,26]$, which make it a suitable candidate region supporting the emergence of behavioral control mediated social norm compliance.

A recent study showed that social norm compliant sharing in children increased between the ages of 6-13 years and this correlated directly with an independent measure of behavioral motor control [27]. Simultaneously recorded brain 
activity showed that this age-related increase in social norm compliance correlated positively with activity in the left DLPFC. Further, the age-dependent increase in activity in this region mediated the developmental increase in behavioral motor control, which in turn predicted the increase in social norm compliance throughout childhood. While connectivity analyses were not conducted in this study, it is likely that such decisions to share are supported by increased functional coupling between DLPFC and brain areas that compute the value of decisions, such as the ventromedial prefrontal cortex (VMPFC). Such a mechanism has been shown to support decisions in favour of long-term goals in similar scenarios in both adults $[20,28,29]$ as well as during middle childhood [30]. This interpretation is buttressed by findings from a recent study measuring event-related potentials (ERPs), which show an increase of regulatory processes in bringing about sharing during childhood [31]. Thus, in older children the P3, a component reflecting behavioral control mechanisms predicted equal sharing of resources, whereas in younger children this was predicted by the EPN, an early component reflecting affective evaluation.

In sum, the development of behavioral control, supported by the maturation of function and connectivity of prefrontal cortical circuitry can account for the observed changes in sharing during middle and late childhood. Such a mechanism helps to shift decisions away from the immediate desires of reward maximization to complying to social norms of equal sharing. 


\section{The neurocognitive mechanisms of helping}

Studies on the ontogeny of prosociality have largely focused on helping, showing that this emerges as early as 14 months [32]. Helping others also implies costs in terms of time, effort and opportunities. Two key motivations have been argued to underlie helping. A selfish desire to reduce one's own distress, also known as personal distress; and an altruistic desire to reduce the other's distress, also known as empathic concern $[33,34]$. Whereas personal distress leads to helping only when there is no other recourse of stopping one's own distress (i.e. fleeing the situation), empathic concern leads to helping across a range of situations. Especially in children, only indicators of empathic concern were shown to predict helping [for a review see 34]. It has been argued that empathic concern arises out of the interplay of an emotional response to the need of another and a sufficiently strong regulation of this emotional response [34]. Thus, the literature on the development of helping during childhood suggests that those children both high in emotional responding and emotion regulation are the ones most likely to help [35]. Support for this comes from studies using parental questionnaires, parent and teacher ratings as well as psychophysiological indicators suggesting that empathic concern is a good predictor of helping behavior in childhood.

In adults it has been shown that observing the painful or unpleasant experience of another person activates circuitry that is also recruited when undergoing this experience oneself $[36,37]$. This circuitry comprises the bilateral anterior insula and medial/anterior cingulate cortex [see Figure 1; 38]. Activity in the anterior insula was shown to correlate positively with empathic concern ratings [36] and 
activity of this region when watching others in pain was positively related to helping behavior, albeit only to in-group members [39]. It has been argued that the anterior insula performs value computations related to prosocial behavior [40]. More recently, it was shown that especially connectivity between the anterior cingulate cortex (ACC) and the anterior insula was positively related to prosocial behavior following an empathy induction [41]. The ACC has been implicated in top-down regulation and control of negative emotions and processing of emotional conflict [42] and in this case might function as affective regulation mechanism to produce empathic concern in turn leading to prosocial behavior.

Several studies on the neurocognitive development of empathic concern and helping have been performed in children. These studies show that children aged 7-12 years activate anterior insula as well as anterior midcingulate when observing the pain of another [43]. Similar activation patterns could be already seen from 4 years of age [44]. A study testing 7-40 year old participants showed that activity in the amygdala in response to seeing others' pain decreased with age, while activity in lateral prefrontal cortex increased, suggesting a potential decrease in distress-related and an increase in emotion regulation related brain functions [45]. A recent longitudinal study in children from 10 to 13 years of age showed that empathic concern at age 10 predicted activation of lateral prefrontal regions, namely the inferior frontal gyrus (IFG), which in turn was linked to helping [46]. Importantly, the IFG activation overlapped with coordinates typically found for cognitively effortful processing. Unpublished data from our lab also shows that age-related changes in empathically driven helping 
were strongly linked to increased connectivity between the right anterior insula and lateral and medial prefrontal cortical areas [47].

In sum, the neurocognitive mechanisms of helping during childhood comprise affective responding to the emotional state of another, as coded in the anterior insula, in combination with regulatory mechanisms of the experienced affect, instantiated in prefrontal cortical brain regions.

\section{Summary}

This review summarizes the recent literature on the neurocognitive mechanisms that support the development of prosocial behavior in childhood. The review draws on a value-based decision-making framework of social behavior [6] and combines this with developmental neuroscience to examine the regulatory mechanisms that predispose children towards prosocial actions in spite of associated costs. One key finding is that neurocognitive mechanisms differ depending on the prosocial behavior in question. Whereas sharing requires behavioral control, helping needs the regulation of emotions in response to another's distress. These distinct mechanisms draw on distinct neural circuitry, which in turn could explain why different types of prosocial behavior correlate so poorly during development [1].

One important feature of prosocial behavior is that it is vastly contextdependent. From around 5 years, children discriminate who they share with based on group membership, familiarity, similarity, their partner's willingness to share, their partner's needs and reputation concerns [for a review see 48]. A 
value-based decision-making framework of prosociality allows ample room for the influence of such contextual variables, since these will affect the value of prosocial behavior depending on the goals of the benefactor.

The present review applies to situations in which prosociality is costly. I contend that even though some experimental settings manage to eliminate the costs of prosocial behavior, the vast majority of real-life situations imply a cost to prosocial behavior. In spite of such costs it has been argued that prosocial behavior does not require any active regulation, but rather occurs effortlessly and automatically [49]. While the idea that prosociality occurs automatically and without any regulation remains contentious [50] the evidence at least during childhood speaks against this. With developmental changes in neurocognitive architecture, it is likely that the mechanisms supporting prosociality change with age, which should preclude definite inferences made from one age group to another.

There is strong evidence that the maturation of prefrontal cortically mediated regulation supports the development of prosocial behavior during childhood. A value-based framework provides a mechanistic account of developmental change in prosocial behavior. Such a framework can simultaneously account for maturational changes in observable behavior and accommodate for the effect of contextual variables likely to influence the computation of costs and benefits associated with the various available social. 


\section{References:}

1. Dunfield, K., et al., Examining the Diversity of Prosocial Behavior: Helping, Sharing, and Comforting in Infancy. Infancy, 2011. 16(3): p. 227-247.

2. Warneken, F., Insight into the biological foundation of human altruistic sentiments. Current Opinion in Psychology, 2016. 7: p. 51-56.

3. Eisenberg-Berg, N., et al., Effects of instructions concering ownership of a toy on preschoolers' sharing and defensive behaviors. Developmental Psychology, 1979. 15: p. 460-461.

4. Svetlova, M., S.R. Nichols, and C.A. Brownell, Toddlers' prosocial behavior: from instrumental to empathic to altruistic helping. Child Dev, 2010. 81(6): p. 1814-27.

5. Blake, P.R. and D.G. Rand, Currency value moderates equity preference among young children. Evolution and Human Behavior, 2010. 31(3): p. 210-218.

6. Ruff, C.C. and E. Fehr, The neurobiology of rewards and values in social decision making. Nat Rev Neurosci, 2014. 15(8): p. 549-62.

7. Paulus, M., et al., Neural correlates of prosocial behavior in infancy: Different neurophysiological mechanisms support the emergence of helping and comforting. Neuroimage, 2012. 66C: p. 522-530.

8. Schmidt, M.F.H. and J.A. Sommerville, Fairness Expectations and Altruistic Sharing in 15-Month-Old Human Infants. Plos One, 2011. 6(10).

9. Geraci, A. and L. Surian, The developmental roots of fairness: infants' reactions to equal and unequal distributions of resources. Dev Sci, 2011. 14(5): p. 1012-20.

*10. McAuliffe, K., et al., The developmental foundations of human fairness. Nature Human Behaviour, 2017. 1(2): p. 0042.

This paper synthesizes a large body of literature on the development of human fairness with an emphasis on the psychological and neural mechanisms

11. Smith, C.E., P.R. Blake, and P.L. Harris, I should but I won't: why young children endorse norms of fair sharing but do not follow them. PLoS One, 2013. 8(3): p. e59510.

*12. Buckholtz, J.W., Social norms, self-control, and the value of antisocial behavior. Current Opinion in Behavioral Sciences, 2015. 3: p. 122-129.

This paper introduces a value-based account of pro- and antisocial behavior

13. Aguilar-Pardo, D., R. Martinez-Arias, and F. Colmenares, The role of inhibition in young children's altruistic behaviour. Cogn Process, 2013. 14(3): p. 301-7.

14. Paulus, M., et al., Social understanding and self-regulation predict preschoolers' sharing with friends and disliked peers: A longitudinal study. International Journal of Behavioral Development, 2015. 39: p. 53-64.

15. Blake, P.R., et al., Prosocial norms in the classroom: The role of selfregulation in following norms of giving. Journal of Economic Behavior and Organization, 2015. 115: p. 18-29.

16. Steinbeis, N., Taxing behavioral control diminishes sharing and costly punishment in childhood. Developmental Science, in press.

*17. Steinbeis, N. and H. Over, Enhancing behavioral control increases sharing in children. J Exp Child Psychol, 2017. 159: p. 310-318. 
*This paper goes beyond correlative measures and reports on the use of an experimental manipulation to demonstrate the importance of behavioral control in sharing during childhood.

18. Spitzer, M., et al., The neural signature of social norm compliance. Neuron, 2007. 56(1): p. 185-196.

19. Ruff, C.C., G. Ugazio, and E. Fehr, Changing social norm compliance with noninvasive brain stimulation. Science, 2013. 342(6157): p. 482-4.

20. Baumgartner, T., et al., Dorsolateral and ventromedial prefrontal cortex orchestrate normative choice. Nature neuroscience, 2011. 14(11): p. 1468U149.

21. Lenroot, R.K. and J.N. Giedd, Brain development in children and adolescents: insights from anatomical magnetic resonance imaging. Neurosci Biobehav Rev, 2006. 30(6): p. 718-29.

22. Lebel, C., et al., Diffusion tensor imaging of white matter tract evolution over the lifespan. Neuroimage, 2012. 60(1): p. 340-52.

23. Hagmann, P., et al., White matter maturation reshapes structural connectivity in the late developing human brain. Proc Natl Acad Sci U S A, 2010. 107(44): p. 19067-19072.

24. Miller, E.K. and J.D. Cohen, An integrative theory of prefrontal cortex function. Annual Review of Neuroscience, 2001. 24: p. 167-202.

25. Rubia, K., et al., Linear age-correlated functional development of right inferior fronto-striato-cerebellar networks during response inhibition and anterior cingulate during error-related processes. Hum Brain Mapp, 2007. 28(11): p. 1163-77.

26. Bunge, S.A., et al., Immature frontal lobe contributions to cognitive control in children: evidence from fMRI. Neuron, 2002. 33(2): p. 301-11.

27. Steinbeis, N., B.C. Bernhardt, and T. Singer, Impulse Control and Underlying Functions of the Left DLPFC Mediate Age-Related and Age-Independent Individual Differences in Strategic Social Behavior. Neuron, 2012. 73(5): p. 1040-1051.

28. Hare, T.A., C.F. Camerer, and A. Rangel, Self-Control in Decision-Making Involves Modulation of the vmPFC Valuation System. Science, 2009. 324(5927): p. 646-648.

29. Hare, T.A., S. Hakimi, and A. Rangel, Activity in dIPFC and its effective connectivity to vmPFC are associated with temporal discounting. Front Neurosci, 2014. 8: p. 50.

30. Steinbeis, N., et al., Development of Behavioral Control and Associated vmPFC-DLPFC Connectivity Explains Children's Increased Resistance to Temptation in Intertemporal Choice. Cereb Cortex, 2016. 26(1): p. 32-42.

*31. Meidenbauer, K.L., et al., A Developmental Neuroscience Study of Moral Decision Making Regarding Resource Allocation. Child Dev, 2016.

This paper reports ERP evidence showing that development of regulatory mechanisms aid the development of sharing during childhood.

32. Warneken, F. and M. Tomasello, Helping and cooperation at 14 months of age. Infancy, 2007. 11(3): p. 271-294.

33. Batson, C.D., The Altruism Question: Toward a Social-Psychological Answer. 1991, Hillsdale, NJ: Erlbaum. 
34. Eisenberg, N., Emotion, regulation, and moral development. Annu Rev Psychol, 2000. 51: p. 665-97.

35. Eisenberg, N., et al., Contemporaneous and longitudinal prediction of children's sympathy from dispositional regulation and emotionality. Developmental Psychology, 1998. 34(5): p. 910-924.

36. Singer, T., et al., Empathy for pain involves the affective but not sensory components of pain. Science, 2004. 303(5661): p. 1157-1162.

37. Wicker, B., et al., Both of us disgusted in My insula: the common neural basis of seeing and feeling disgust. Neuron, 2003. 40(3): p. 655-64.

38. Lamm, C., J. Decety, and T. Singer, Meta-analytic evidence for common and distinct neural networks associated with directly experienced pain and empathy for pain. Neuroimage, 2011. 54(3): p. 2492-2502.

39. Hein, G., et al., Neural responses to ingroup and outgroup members' suffering predict individual differences in costly helping. Neuron, 2010. 68(1): p. 149-60.

40. Hare, T.A., et al., Value Computations in Ventral Medial Prefrontal Cortex during Charitable Decision Making Incorporate Input from Regions Involved in Social Cognition. Journal of Neuroscience, 2010. 30(2): p. 583590.

41. Hein, G., et al., The brain's functional network architecture reveals human motives. Science, 2016. 351(6277): p. 1074-8.

42. Etkin, A., T. Egner, and R. Kalisch, Emotional processing in anterior cingulate and medial prefrontal cortex. Trends Cogn Sci, 2011. 15(2): p. 85-93.

43. Decety, J., K.J. Michalska, and Y. Akitsuki, Who caused the pain? An fMRI investigation of empathy and intentionality in children. Neuropsychologia, 2008. 46(11): p. 2607-2614.

44. Michalska, K.J., K.D. Kinzler, and J. Decety, Age-related sex differences in explicit measures of empathy do not predict brain responses across childhood and adolescence. Developmental Cognitive Neuroscience, 2013. 3: p. 22-32.

45. Decety, J. and K.J. Michalska, Neurodevelopmental changes in the circuits underlying empathy and sympathy from childhood to adulthood. Developmental Science, 2010. 13(6): p. 886-899.

**46. Flournoy, J.C., et al., Neural Reactivity to Emotional Faces May Mediate the Relationship Between Childhood Empathy and Adolescent Prosocial Behavior. Child Dev, 2016. 87(6): p. 1691-1702.

This paper reports unique longitudinal data on the neurocognitive mechanisms of helping in childhood.

47. Hoffmann, F., et al., Maturation of prefrontal-insular connectivity predicts developmental shift towards altruistic helping in childhood. in preparation.

48. Martin, A. and K.R. Olson, Beyond good and evil: what motivations underlie children's prosocial behavior? Perspect Psychol Sci, 2015. 10(2): p. 15975.

49. Rand, D.G., J.D. Greene, and M.A. Nowak, Spontaneous giving and calculated greed. Nature, 2012. 489(7416): p. 427-430.

50. Krajbich, I., et al., Rethinking fast and slow based on a critique of reactiontime reverse inference. Nat Commun, 2015. 6: p. 7455. 


\section{Acknowledgements:}

This research is funded by an ERC Starting Grant for Innovative Ideas (ERC2016-StG-715282) and a Jacobs Foundation Early Career Research Fellowship.

\section{Figures}

Figure 1. Overview of brain regions involved in prosocial behavior during childhood A. Lateral slice showing anterior insula (red) and dorsolateral prefrontal cortex (purple). B. Medial slice showing anterior cingulate (red) and ventromedial prefrontal cortex (blue). 